\title{
Classification of Colorectal Cancer Based on Molecular Features
}

\section{Behzad hatami, Ehsan Nazemalhosseini mojarad, Roya Kishani Farahani*}

${ }^{1}$ Basic and Molecular Epidemiology of Gastrointestinal Disorders Research Center, Research Institute for Gastroenterology and Liver Disease, Shahid Beheshti University of Medical Sciences, Tehran, Iran

${ }^{2}$ Gastroenterology and Liver Disease Research Center, Research Institute for Gastroenterology and Liver Disease, Shahid Beheshti University of Medical Sciences, Tehran, Iran

\section{Editorial}

Colorectal cancer (CRC) is a major cause for morbidity and mortality worldwide; it is the fourth most common cancer in men and the third most common cancer in women [1]. CRC is divided into three categories: hereditary, nonhereditary and familial $[2,3]$. Approximately $15 \%$ of CRC cases are considered as a hereditary form which most common contains: Familial adenomatous polyposis (FAP) and hereditary non polyposis colorectal cancer (HNPCC) and MYHAssociated polyposis (MAP) [4,5]. CRC develops through multiple pathways leading to dL gerent phenotypes. Hese pathways may be defined on molecular features: 1) chromosomal instability (CIN), Microsatellite instability (MSI) and $\mathrm{CpG}$ island methylator phenotype (CIMP) [6]. CIN, or classic adenoma-to-carcinoma pathway, account $65-70 \%$ of sporadic CRC, is characterized by an imbalance in chromosome number (aneuploidy), chromosomal genomic amplLficatLons, and a high frequency of $\mathrm{LOH}$, which has been determined through a series of mutations in tumor suppressor genes or oncogenes, in some pathways including: WNT/APC/B-CAT, RAS, P53, PI3KCA pathway. 18q LOH where the genes Smad2, Smad4 and DCC are located and also loss of $8 \mathrm{p}$ and $5 \mathrm{q}$ allele correlated with CIN pathway [7-10]. Recently, mentioned hypomethylation of LINE -1 is also associated with the CIN pathway [11]. CIN-positive tumors are generally associated with poor prognosis, distally located and tend to be well- or moderately dLg gerentLated [12]. MSI is observed in $15 \%$ of CRCs and also most of these tumors are sporadic which is caused by defection of the DNA Mismatch Repair (MMR) system containing MLH1, PMS2, MSH6, or MSH2 genes. MSI status grouped as: MSI high (MSI-H), MSI low (MSL-L) and MS stable (MSS). MSI tumors present particular clinical features: proximal located, poorly $\mathrm{dL}$ gerentLated a mucinous or medullary histotype and oien presents intense peritumoral and intratumoral lymphocytic LnfiltratLons [13,14]. MSI-high CRC does not respond to -fluorouracll-based chemotherapies and frequently harbor the BRAF V600E mutation [13,15]. More than $80 \%$ of MSICRC harbor mutations of the TGF- $\beta$ Receptor II (TGF- $\beta$ RII) [15]. In general, the prognosisand survival of MSI-high CRC patients is better than CIN positive CRC patients [14]. He last pathway is characterized by epigenetic alterations, resulting in aberrant hypermethylation of $\mathrm{CpG}$ dinucleotide sequences located in the promoter regions of genes involved in cell cycle regulation, apoptosis, angiogenesis, DNA repair, invasion and adhesion. It is found in approximately $20-30 \%$ of CRC [7]. Clinical features of CIMP CRCs are similar to those associated with MSI [16]. Ogino et al. suggested using eight markers (CACNA1G, p16CDKN2A, CRABP1, IGF2, hMLH1, NEUROG1, RUNX3, and SOCS1) to classLficatLon CRC subgroups if 1 to 5 out of 8 markers methylated known as CIMP-low, when none of each markers methylated means CIMP- 0 , and 6 to 8 out of 8 markers have promoters methyled are - CIMP-high[16]. Hese three CRC pathways are not mutually exclusive, with some tumors exhibiting features of more than one pathway [10]. Based on, simultaneous presence of multiple pathways in tumors, there are five molecular CRC subtypes, with $\mathrm{dL}$ gerent molecular profile and clinicpathological features including: 1) CIMP high, MLH1 methylation, MSI high, Braf mutation; (account for $12 \%$ of CRC). 2) CIMP high, BRAF mutation, methylation of multiple genes, MSI low or microsatellite stable; (8\%). 3) CIMP negative, K-ras mutation, MGMT methylation, CIN, MSI low or microsatellite stable; (20\%). 4) CIMP negative, CIN, microsatellite stable; (57\%). 5) CIMP negative, MSI high; negative for BRAF mutations (HNPCC) [10]. In conclusion, Molecular classification of CRC is suitable for better understanding of mechanism involved in initiation and development of CRC. Since CRC subtypes have distinct prognosis, chemosensitivity and different survival, these classLficatLons can provide a better guide for patient stratLficatLon in order to ultimately personalized medicine to improve effective treatments.

\section{References}

1. Parkin DM, Bray F, Ferlay J, Pisani $P$ (2002) Global cancer statistics. CA Cancer J Clin 55: 74-108

2. Snover DC (2011) Update on the serrated pathway to colorectal carcinoma Hum Pathol 42: 1-10.

3. Half E, Bercovich D, Rozen P (2009) Familial adenomatous polyposis Orphanet J Rare Dis 4: 22.

4. Bishop JM (1987) The molecular genetics of cancer. Science 235: 305-311.

5. Knudson AG (1985) Hereditary cancer, oncogenes, and antioncogenes. Cancer Res 45: 1437-1443.

6. Kalady MF, Dejulius KL, Sanchez JA, Jarrar A, Liu X, et al. (2012) BRAF mutations in colorectal cancer are associated with distinct clinical characteristics and worse prognosis. Dis Colon Rectum 55: 128-133.

7. Colussi D, Brandi G, Bazzoli F, Ricciardiello L (2013) Molecular Pathways Involved in Colorectal Cancer:Implications for Disease Behavior and Prevention. Int. J. Mol. Sci 14: 16365-16385

8. Baker SJ, Preisinge AC, Jessup JM, Paraskeva C, Markowitz S, et al. (1990) p53 gene mutations occur in combination with $17 \mathrm{p}$ allelic deletions as late events in colorectal tumorigenesis. Cancer Res 50: 7717-7722.

9. Kern SE, Fearon ER, Tersmette KW, Enterline JP, Leppert M, et al. (1989) Hamilton SR. Clinical and pathological associations with allelic loss in colorectal carcinoma [corrected]. JAMA 261: 3099-3103.

10. Al-Sohaily S, Biankin A, Leong R, Kohonen-Corish M, Warusavitarne J (2012) Molecular pathways in colorectal cancer. Journal of Gastroenterology and Hepatology 27: 1423-1431.

11. Baba Y, Huttenhower C, Nosho K, Tanaka N, Shima K, et al. (2010) Epigenomic diversity of colorectal cancer indicated by LINE-1 methylation in a database of 869 tumors. Mol.Cancer 9: 125.

12. Domingo E, Ramamoorthy R, Oukrif D, Rosmarin D, Presz M, et al. (2013) Use of multivariate analysis to suggest a new molecular classification ofcolorectal cancer. J Pathol 229: 441-448.

13. Sinicrope FA, Sargent DJ (2012) Molecular pathways: Microsatellite instability

*Corresponding author: Roya Kishani Farahani, Basic and molecular epidemiology of Gastrointestinal disorders Research center, Research Institute for Gastroenterology and Liver Disease, Shahid Beheshti University of Medical Sciences, Tehran, Iran, Tel: +9829902233; E-mail: Roya.kishani@yahoo.com

Received June 18, 2015; Accepted August 10, 2015; Published August 12, 2015

Citation: Hatami B, Mojarad EN, Farahani RK (2015) Classification of Colorecta Cancer Based on Molecular Features. Gene Technology 4: e115. doi: 10.4172/2329$6682.1000 \mathrm{e} 115$

Copyright: $\odot 2015$ Hatami B, et al. This is an open-access article distributed under the terms of the Creative Commons Attribution License, which permits unrestricted use, distribution, and reproduction in any medium, provided the original author and source are credited. 
Citation: Hatami B, Mojarad EN, Farahani RK (2015) Classification of Colorectal Cancer Based on Molecular Features. Gene Technol 4: e115. doi: 10.4172/2329-6682.1000e115

in colorectal cancer,prognostic, predictive, and therapeutic implications. Clin. Cancer Res 18: 1506-1512.

14. Lanza G, Gafa R, Maestri I, Santini A, Matteuzzi M, et al. (2002) Immunohistochemical pattern of MLH1/MSH2 expression is related to clinical and pathological features in colorectal adenocarcinomas with microsatellite instability. Mod. Pathol 15: 741-749.

15. Parsons MT, Buchanan DD, Thompson B, Young JP, Spurdle AB (2012) Correlation of tumour BRAF mutations and MLH1 methylation with germline mismatch repair (MMR) gene mutation status: A literature review assessing utility of tumour features for MMR variant classification. J Med Genet 49: 151-157.

16. Nazemalhosseini Mojarad E, Kuppen JK, Asadzadeh P, Aghdaei H, Zali MR (2013) The CpG island methylator phenotype (CIMP) in colorectal cancer Gastroenterol Hepatol Bed Bench 6: 120-128. 